\title{
Music of grain crushing with the Discrete Element Method
}

\author{
Li Zeng ${ }^{1}$, Andres Alfonso Peña Olarte ${ }^{1 *}$, and Roberto Cudmani ${ }^{1}$ \\ ${ }^{1}$ Zentrum Geotechnik, Technical University of Munich, Franz-Langinger-Str. 10, 81245 Munich, Germany
}

\begin{abstract}
A series of compression tests on agglomerates of microspheres representing a single grain are conducted to investigate the impact of heterogeneity on the acoustic emissions (AE) generation. The grain heterogeneity is realized by using a Weibull shape parameter-augmented traditional discrete element method (DEM). During the compression process the development of the micro-cracks, and the magnitude and location of the AE events are tracked and recorded. Through a 3D visualization of the AE events, their location and the clustered broken bonds are identified. The current study demonstrates the potential of AE measurements to track changes in the fabric and structure of granular materials. The results of this DEM study will contribute to clarify the mechanism of particle breakage and its consideration in practical applications.
\end{abstract}

\section{Introduction}

Particle breakage is very relevant for a clear understanding of the mechanical behaviour of sands and is therefore important to many problems in geotechnical engineering practice 0 . Grain crushing impacts not only the stress-strain behaviour of granular materials, but also its permeability by altering the grain size distribution 0 . Over the last several decades, a number of experimental studies have been conducted with the aim of enhancing the understanding of grain breakage [3-6]. However, due to the difficulties in observing the evolution of grain crushing in experiments, its effect on the mechanical behaviour is not yet well-understood [7, 8] and rarely take into account in geotechnical engineering practice. Moreover, so far limited numerical models can capture realistically the heterogeneity of the grain structure to reflect the fracturing and crushing process of natural grains 0 .

The discrete element method (DEM) proposed by Cundall and Strack 0 offers an efficient approach to modelling the entire evolution of grain crushing from macro and micro scale perspectives for crushable materials, which could resolve the limitations of laboratory tests.0.

Acoustic Emissions are a non-destructive observation of energy release prior, during, and after fracture of brittle materials 0 . Part of the energy dissipated during this permanent deformation of particulate materials is converted to heat and sound. The high-frequency portion of this sound energy $(>10 \mathrm{kHz})$ is known as acoustic emission (AE) 0. It refers to the transient elastic waves generated by the rapid release of energy from a material, signalling the process of material plastic deformation 0 .

In this contribution, the coupling of a heterogeneity model, a DEM model and an acoustic emission model is used to investigate in agglomerate compression test, the effect of grain heterogeneity on pre- and post-fracture behaviour and related AE events. In Section 2, we briefly describe the numerical model. The results of the analyses and the conclusion are presented in Section 3 and Section 4, respectively.

\section{Numerical model of compression test}

The DEM simulations of the agglomerate compression test are carried out using YADE, which is an extensible open-source framework for discrete numerical models 0 . This study adopted the construction algorithms, which create a sample by placing each and every element exclusively based on boundary geometry defined by a sphere.

Fig. 1 presents the simulation set-up of the agglomerate experiment. Thereby, an agglomerate is compressed vertically between two flat rigid plates while the displacement of the plates and the applied force are recorded. The upper platen moves slowly downward. A loading rate of $0.001 \mathrm{~m} / \mathrm{s}$ was selected to reduce computational cost while maintaining an inertial number well below the "critical" threshold 0 . The distance between the two loading platens is $48 \mathrm{~mm}$ (agglomerate diameter) and the corresponding strain rate is $0.208 \mathrm{~s}^{-1}$. Different rates are used to check the influence on the system response.

A cohesive frictional behaviour of the grain is assumed, and the Yade's DEM-Bonded Particle Model (BPM) formulation is used 0 . The contact between two particles had 3 degrees of freedom (1 normal and 2 shear components), including cohesive and frictional parameters 0 . A linear contact model is used to evaluate the contact forces:

$$
\boldsymbol{f}_{i j}^{n}=k_{i j}^{n} \cdot \Delta D_{i j} \cdot \boldsymbol{n}_{i j}^{n}
$$

\footnotetext{
* Corresponding author: andres.pena@tum.de

A video is available at https://doi.org/10.48448/3yk1-vr07
} 
where $\boldsymbol{f}_{i j}^{n}$ is the normal force applied to the interacting particles $i$ and $j, \Delta D_{i j}=D_{i j}-D_{e q}$ is the difference between the displacement between the interacting particles and the predefined equilibrium distance. $\boldsymbol{n}_{i j}^{\boldsymbol{n}}$ is the unit vector parallel to the interaction. The spring normal stiffness $k_{i j}^{n}$ is determined on the assumption that two springs are in series with lengths equal to the interacting particle radii:

$$
k_{i j}^{n}=\frac{E_{i} r_{i} E_{j} r_{j}}{E_{i} r_{i}+E_{j} r_{j}},
$$

where $\mathrm{E}$ is microscopic Young's modulus of particle $i$ or $j$. The maximum tensile force is described as:

$$
\left\|\boldsymbol{f}^{n}\right\|_{\max }=-t \cdot A_{\text {int }}
$$

where $t$ is the tensile strength of the bond and $A_{\text {int }}=\pi$. $\min \left(r_{i}, r_{j}\right)^{2}$.

Maximum allowable shear force $\left\|\boldsymbol{f}^{t}\right\|_{\max }$ is defined by the normal force $\left\|\boldsymbol{f}^{n}\right\|$, the cohesion $\mathrm{c}$, the local friction angle $\varphi_{b}$. The maximum shear force is calculated for a bonded interaction according to:

$$
\left\|\boldsymbol{f}^{\boldsymbol{t}}\right\|_{\max }=\left\|\boldsymbol{f}^{\boldsymbol{n}}\right\| \tan \varphi_{b}+c A_{\text {int }} .
$$

By assuming that each broken DEM bond (or cluster of broken bonds) represents an event position, $\mathrm{AE}$ events are numerically simulated, while the corresponding cluster strain energy change is empirically related to the magnitude of the event $[11,14]$. In real tests, $\mathrm{AE}$ can also arise from frictional processes, though when it comes to single grain crushing, the contribution of friction is limited and thus not considered in this work. Once a bond breaks, the strain energies $\left(u_{i}\right)$ are summed up within a predefined volume in terms of particle diameters $(\lambda)$ for all intact bonds:

$$
u_{i}=\frac{1}{2}\left(\frac{f_{n}^{2}}{k_{n}}+\frac{f_{s}^{2}}{k_{s}}\right),
$$

where $U_{0}=\sum_{i=1}^{n} u_{i}$ is the reference strain energy, $n$ is the number of interactions within the particle neighbourhood, $f_{n}, f_{s}$ and $k_{n}, k_{s}$ are the normal and shear force $(\mathrm{N})$ and stiffness $(\mathrm{N} / \mathrm{m})$ components of the interaction prior to failure, respectively. Additionally, the associated system strain energy change represents the event magnitude. The empirical equation used to calculate the moment magnitude $M_{e}$ of the $\mathrm{AE}$ event is as follows 0 :

$$
M_{e}=\frac{2}{3} \log \Delta U-3.2,
$$

where $\Delta U$ is the change value of strain energy in the associated system (Joules). After initial breakage, $\Delta U$ is computed for each subsequent time step as $\Delta U=U_{0}$ $\sum_{i=1}^{n} u_{i}$. The location of a clustered event is simply the average of the clustered event centroids 0 .

A time phase window $\left(T_{\max }\right)$ based on the P-Wave velocity associated with interacting particle densities is defined as 0 :

$$
T_{\text {max }}=\operatorname{int}\left(\frac{D_{a v g} \lambda}{\max \left(v_{p 1}, v_{p 2}\right) \Delta t}\right),
$$

where $D_{\text {avg }}$ is the average diameter of the particles comprising the failed event $(\mathrm{m}), v_{p}$ is the P-Wave velocity of interacting particles $(\mathrm{m} / \mathrm{s}), \Delta \mathrm{t}$ is the time step of the simulation (seconds/time step), and $\lambda$ is the moment radius factor. Events are clustered if they occur within temporal $T_{\max }$ and spatial window $\lambda$ of other events 0 . The DEM simulation parameters are presented in Table 1. The agglomerate parameters are selected from the values presented in 0 , where a DEM model is calibrated to the laboratory tests of single grain compression of rock pebbles.

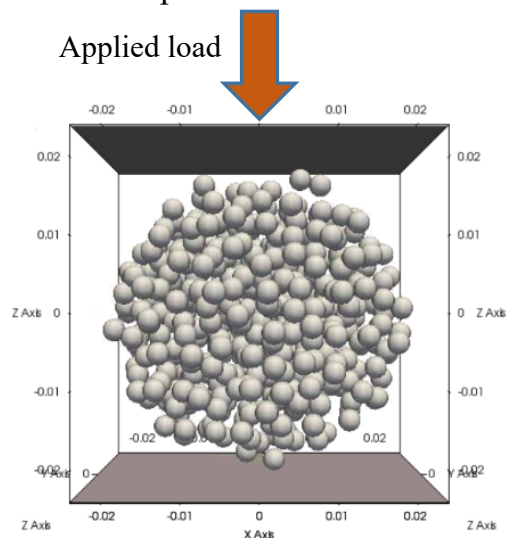

Fig. 1. Numerical specimen of agglomerate under compression (number of particles 448).

Our simulations aim to demonstrate the influence of the Weibull shape parameter, represented by $\gamma$, on the spatial and load-based distribution of simulated $\mathrm{AE}$ activity in the agglomerate DEM model. The Weibull shape parameter $\gamma$ controls the heterogeneity of the tensile strength of the bonds, which influence the $\mathrm{AE}$ events during the compression of the agglomerate. The smaller the $\gamma$ value, the greater the heterogeneity of the tensile strength 0 . In this paper, we run a simulation of a homogeneous model $(\gamma=\infty)$ and compare it with three simulations of various heterogeneity models $(\gamma=2,4$, 6). The void fraction of the agglomerate is 0.85 .

Table 1. DEM simulation parameters

\begin{tabular}{|c|c|c|}
\hline \multicolumn{2}{|c|}{ Micro parameter } & Value \\
\hline Radius of the agglomerate & $\mathrm{R}$ & $24 \mathrm{~mm}$ \\
\hline Mean radius of particles & $\mathrm{r}$ & $1.65 \mathrm{~mm}$ \\
\hline Density & $\rho$ & $2706 \mathrm{~kg} / \mathrm{m}^{3}$ \\
\hline Effective modulus & $\mathrm{E}$ & $40 \mathrm{GPa}$ \\
\hline Stiffness ratio & $\mathrm{k}_{\mathrm{s}} / \mathrm{kn}_{\mathrm{n}}$ & 0.4 \\
\hline Friction coefficient & $\mathrm{u}$ & 0.7 \\
\hline Cohesion strength & $\mathrm{c}$ & $35 \mathrm{MPa}$ \\
\hline Tensile strength & $\mathrm{t}$ & $35 \mathrm{MPa}$ \\
\hline Weibull shape parameter & $\gamma$ & $2,4,6, \infty$ \\
\hline Moment radius factor & $\lambda$ & 4 \\
\hline
\end{tabular}

\section{Results}

\subsection{Load-deflection response}

The results of the four simulations are presented in Fig. 2. To analyze the shown load vs deflection curves, we calculate the overall stiffness $\mathrm{k}$ of the grain as:

$$
k=F / d,
$$

where $\mathrm{F}$ is the $50 \%$ of the maximum load (failure load) and $\mathrm{d}$ is the corresponding deflection. For $\gamma=2$ we get the min stiffness $\mathrm{k}=34740 \mathrm{kN} / \mathrm{m}$ and for $\gamma=4,6, \infty$, the values of $\mathrm{k}$ are $36158 \mathrm{kN} / \mathrm{m}, \quad 35754 \mathrm{kN} / \mathrm{m}$ and 
$36021 \mathrm{kN} / \mathrm{m}$ respectively. This reveals that $\gamma$ have a negligible influence on the overall stiffness of the specimen for deformations below $50 \%$ of the failure load.

Fig. 3 depicts a relation between the load, AE count and time. It can be clearly seen from the AE count vs times that for the simulation with $\gamma=2$ the AE count increases rapidly with the increase of load, and the load-deflection response deviates from a linear behaviour at about 50\% of failure load. For $\gamma$ values larger than 2 the rate of $\mathrm{AE}$ events slows down, but increases steadily until failure for all cases. These results highlight the significant impact of grain heterogeneity on AE count. The larger the heterogeneity, the larger the number of AE events. This is a result of more bonds failing as bond strength variability increases.

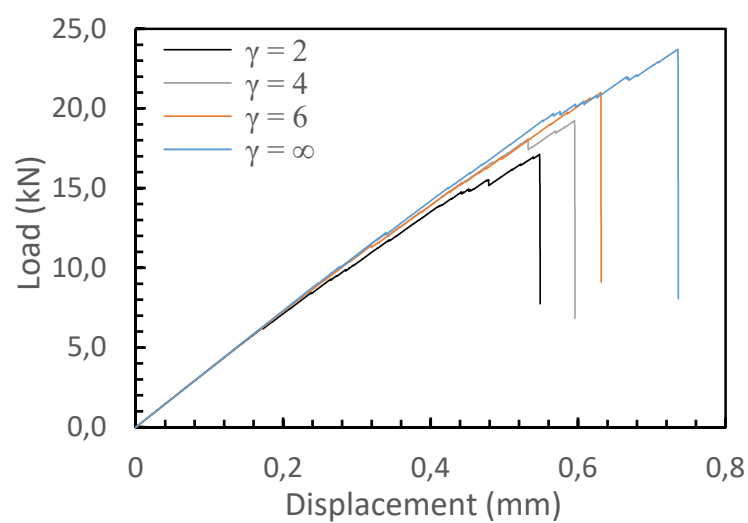

Fig. 2. Load as a function of deflection from agglomerate tests for various Weibull shape parameter $\gamma$.

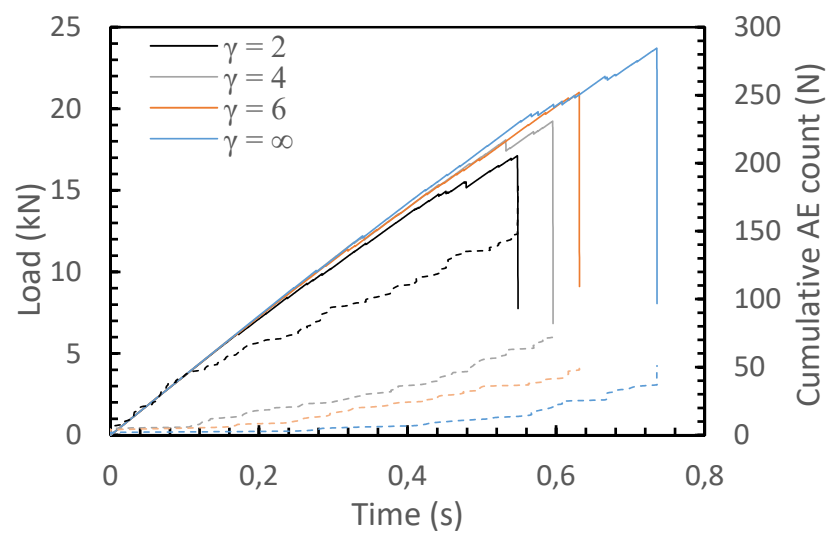

Fig. 3. Load and AE count as a function of time from agglomerate tests.

\subsection{Acoustic emissions}

Each single acoustic emission in the DEM model is comprised of a cluster of broken bonds that occur within a scale of space and time 0 . The temporal evolution of the AE events is stored and visualized using Paraview (open-source, multi-platform data analysis and visualization application 0). Fig. 4 presents snapshots of the numerical AE events and their clustered interactions during the compression for different Weibull shape parameter.

Fig. 4a presents AE events comprised of clustered broken bonds for $\gamma=\infty$. As compared with Fig. 4c, which presents AE events for $\gamma=2$, the numerical homogenous specimen $\gamma=\infty$ exhibits fewer AE events and more localized than $\gamma=2$. Thus, by increasing heterogeneity of the agglomerate specimen, the number of AE events increases significantly, but also the spatial distribution is dispersed over the full specimen.

The cumulative number of AE events vs their magnitude is shown in Fig. 5. The average values of the magnitudes for $\gamma=2$ (183 AE events), 4 (73 AE events), 6 (49 AE events), $\infty$ (51 AE events) are -10.1081, -9.4709, 8.3876 , -7.7433, respectively. The larger the heterogeneity, the smaller the average and the maximum AE magnitudes values.
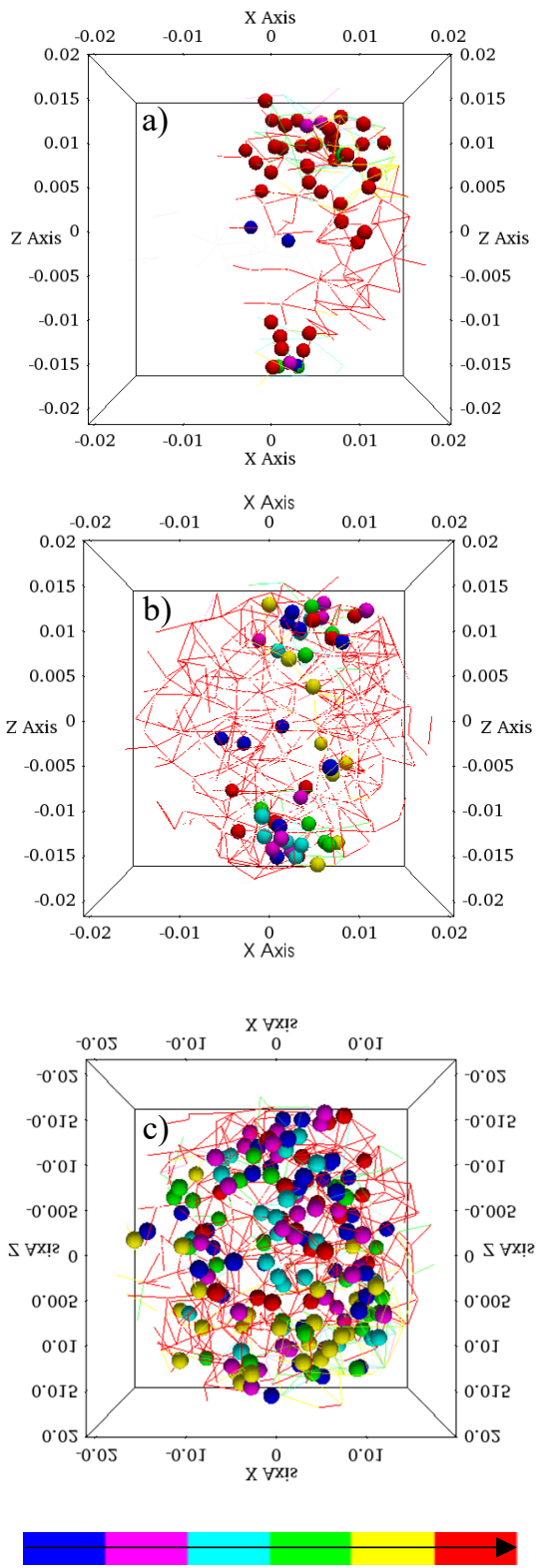

Fig. 4. AE events (sphere) comprised of clustered broken bonds (line) for a value of $60 \%$ maximum load. Weibull shape parameter $\gamma=a) \infty$, b) 6 , c) 2, Colours represent event ID.

The homogenous specimen exhibited the sharpest and largest release of energy through fewer events. The bvalues of the Guttenberg-Richter law are calculated and they vary between 0.5 and 0.7 being at the lower end of expected values. These b-values apply for the range of higher magnitudes between -8 to -5 . 


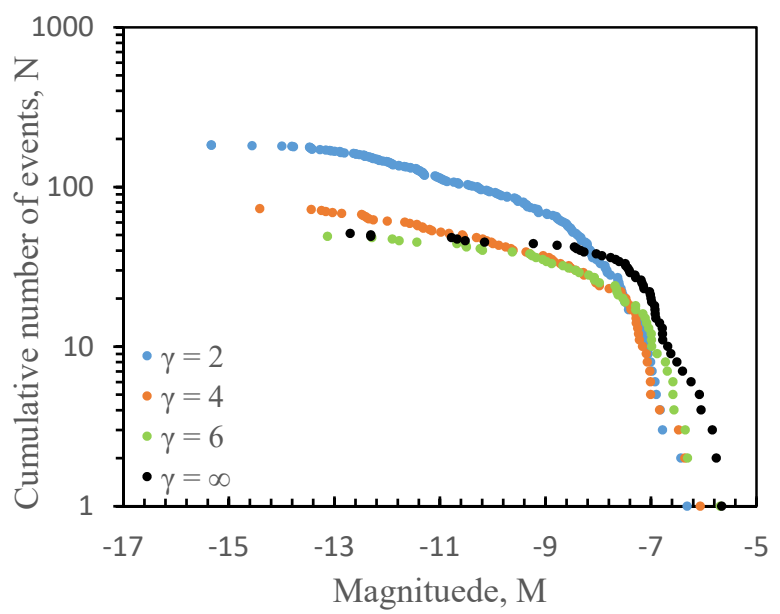

Fig. 5. Acoustic Event moment magnitude distribution for various Weibull shape parameter, $\gamma$.

\section{Conclusions}

A set of agglomerate compression tests is conducted on agglomerate specimens to investigate the impact of heterogeneity on acoustic emissions (AE) generation. For gaining insights into the effect of heterogeneity on the agglomerate fracture process, this study combines conventional DEM with a Weibull distribution controlled by the shape parameter $\gamma$. The $\gamma$-augmented DEM generally represents heterogeneities by stochastically assigning micro-properties (interaction stiffness or strength properties) 0 .

To observe the development of the micro-cracks during the compression process more clearly, the magnitude and location of AE events (clustered broken bonds) is recorded and visualized. We visualize acoustic emission to analyse data qualitatively. The results underline the potential of using AE as a diagnostic tool to study micromechanical behaviour in granular material 0 .

The only variable considered in the simulations is the Weibull shape parameter as indicator of heterogeneity of the grain. Four simulations for various Weibull shape parameter $(\gamma=2,4,6, \infty)$ are used in this study. The results show that the larger the heterogeneity:

- the larger the rate and the number of AE events, and their distribution over the entire specimen,

- the lower the grain strength and

- the smaller the average and the maximum AE magnitude values.

In conclusion, these results are the starting point to analyse and quantify the influence of heterogeneity on crushing and fracturing mechanisms of an agglomerate. It is shown, that heterogeneity is an important factor influencing the AE events.

The results of this DEM study are valuable for the clarification of the mechanism of particle breakage and development of further AE applications. This numerical model provides a method to understand and quantify soil behaviour from AE measurements. Ongoing works focus on investigating the influence of loading rate, the cluster size $(\lambda)$, the number of particles and fracture propagation in the agglomerate model. Future work will address the execution of laboratory element test to validate the numerical model and to gain new insights on ageing phenomena of granular materials.

\section{References}

[1] B. Zhao, J. Wang, M.R. Coop, G. Viggiani, M. Jiang, Géotechnique 65, 8 (2015)

[2] O.H. Al Hattamleh, H.H. Al-Deeky, M.N. Akhtar, Int. J. Geosciences 4, 1055-1060 (2013)

[3] W. Choi, Y. Son, J. Park, S. Noh, T. Bong, An investigation on crushing and particle size distribution characterizes of bottom ash as compaction energy, in 2013 World of Coal Ash (WOCA) Conference, 22-25 April 2013, Lexington, United States (2013)

[4] M.S. Lim, D.C. Wijeyesekera, A. Zainorabidin, I. Bakar, Int. J. Integr. Engineering 4, 4 (2012)

[5] H. Shahnazari, R. Rezvani, Eng. Geol. 159, 98-105 (2013)

[6] R. Russell, I. Einav, Granul. Matter 15, 3 (2013)

[7] J. Lin, E. Bauer, W. Wu, Geosci. Front. 11, 451459 (2020)

[8] J.F. Labuz, L. Biolzi, Int. J. Rock Mech. Min. 44, 525- 537 (2007)

[9] Q. Huang, W. Zhou, G. Ma, T. Ng, K. Xu, Geosci. Front. 11, 401-411 (2020)

[10] P.A. Cundall, O.D.L. Strack, Géotechnique 29, 4765 (1979)

[11] Y. Xiao, C.S. Desai, A. Daouadji, A.W. Stuedlein, H. Liu, H. Abuel-Naga, Geosci. Front. 11, 363-374 (2020)

[12] R.A. Caulk, Open Geomechanics 2, 2 (2020)

[13] A. Smith, N. Dixon, Géotechnique 69, 12 (2019)

[14] W. Mao, Y. Yang, W. Lin, S. Aoyama, I. Towhata, Int. J. Geomech. 18, 11 (2018)

[15] V. Šmilauer et al., Yade Documentation 2nd ed. (2015)

[16] P. Jin, L. Xie, L. Zheng, X. Liang, Y. Li, Analysis on layered rock cutting process with cutter suction dredger based on discrete element method, in 38th IAHR World Congress, 1-6 September 2019, Panama City, Panama (2019)

[17] C.H. Scholz, Harris, R.A. Seismol. Res. Lett. 74, 333-333 (2003)

[18] C. Khazaei, J. Hazzard, R. Chalaturnyk, Acta Geotech. 11, 1351-1367 (2016)

[19] J.F. Hazzard, B. Damjanac, Further investigations of microseismicity in bonded particle models, in 3rd International FLAC/DEM Symposium, 22-24 October 2013, Hangzhou, China (2013)

[20] J. Ahrens, B. Geveci, C. Law, ParaView: An enduser tool for large-data visualization. In Visualization Handbook (2005)

[21] G. Michlmayr, D. Or, Granul. Matter 16, 627-640 (2014) 